\title{
Fibroblast growth factor receptor 1 antagonism attenuates lipopolysaccharide-induced activation of hepatic stellate cells via suppressing inflammation
}

\author{
DAYONG LOU ${ }^{1 *}$, JIBO HAN $^{2 *}$, LIQIN ZHOU ${ }^{1}$, HUANJIE MA ${ }^{1}$, JIANJIANG XV ${ }^{2}$, \\ JUNWEI SHOU $^{1}$, ZHIXIU XU ${ }^{1}$, LIQIN JIANG ${ }^{2}$ and YUANYUAN QIAN ${ }^{1}$ \\ ${ }^{1}$ Medication Department, Zhuji People's Hospital of Zhejiang Province, Zhuji, Shaoxing, Zhejiang 311800; \\ ${ }^{2}$ Department of Cardiology, The Second Affiliated Hospital of Jiaxing University, Jiaxing, Zhejiang 314000, P.R. China
}

Received March 16, 2018; Accepted July 27, 2018

DOI: $10.3892 /$ etm.2018.6586

\begin{abstract}
Activated hepatic stellate cells (HSCs) serve key roles in hepatic fibrosis by producing excessive extracellular matrix (ECM) components. Lipopolysaccharide (LPS) has been found to be associated with hepatic fibrogenesis through direct interactions with HSCs. Recently, the fibroblast growth factor receptor 1 (FGFR1) signalling system was identified as a key player in the process of liver fibrosis. In the present study it was evaluated whether FGFR1 mediated LPS-induced HSCs activation. In cultured cells, FGFR1 was inhibited by either siRNA silencing or by a small-molecule inhibitor in LPS-stimulated HSCs. The blockade of FGFR1 decreased LPS-induced nuclear factor- $\mathrm{kB}(\mathrm{NF}-\mathrm{\kappa B})$ activation, inflammatory cytokine release, fibrosis, and cell proliferation in HSCs. It was further indicated that LPS triggered FGFR1 phosphorylation via TLR4/c-Src. These findings confirmed the detrimental effect of FGFR1 activation in the pathogenesis of LPS-related HSC activation and revealed that FGFR1 may be an ideal therapeutic target for LPS-induced liver fibrosis.
\end{abstract}

\section{Introduction}

Hepatic fibrosis is a wound-repairing response to frequent and repeated liver injuries that may lead to cirrhosis and even

Correspondence to: Dr Liqin Jiang, Department of Cardiology, The Second Affiliated Hospital of Jiaxing University, 1518 Huancheng North Road, Jiaxing, Zhejiang 314000, P.R. China E-mail: lqjiangjx2y@163.com

Dr Yuanyuan Qian, Medication Department, Zhuji People's Hospital of Zhejiang Province, Taozhu Street, 9 Jianmin Road, Zhuji, Shaoxing, Zhejiang 311800, P.R. China

E-mail: m15068255906@163.com

${ }^{*}$ Contributed equally

Key words: fibroblast growth factor receptor 1, liver fibrosis, hepatic stellate cell, lipopolysaccharide, inflammation liver cancer (1). Liver fibrosis represents the consequence of an imbalance between accumulation and dissolution of excessive extracellular matrix (ECM) $(2,3)$, and the inhibition of collagen generation is an effective method to treat liver fibrosis (4,5). Activated hepatic stellate cells (HSCs) are well known for their potential role in increasing deposition of ECM and elevated proliferation in liver fibrosis $(6,7)$. During liver injury, quiescent HSCs that store vitamin A in lipid droplets (LDs) and reside in the spaces of Disse convert to an activated phenotype and are depleted of vitamin A (6). The activation process of quiescent HSCs can be driven by various stimuli, including lipopolysaccharide (LPS) (8). LPS, the classic ligand for Toll-like receptor 4 (TLR4) (9), has been found to be associated with hepatic fibrogenesis through direct interactions with HSCs (8). LPS-induced nuclear factor- $\kappa \mathrm{B}(\mathrm{NF}-\kappa \mathrm{B})$ activation and release of inflammatory cytokines were also observed in activated HSCs (10). However, the molecular mechanisms underlying the effects of LPS on HSC activation are poorly understood.

Fibroblast growth factor receptor 1 (FGFR1) is a receptor tyrosine kinase that mediates a broad spectrum of cellular and developmental processes, including apoptosis, proliferation, and angiogenesis (11). Moreover, substances targeting FGFR1 have been shown to have promise for treatment in animal cancer models (12). Recently, the FGFR1 signalling system has also been identified as a key player in the process of liver injury (13-15). Selective blockade of FGFR1 inhibited HSC activation by measuring the production of ECM (14). In addition, administration of NP603, a novel inhibitor of FGFR1, significantly decreased hepatic fibrosis in carbon tetrachloride (CCl4)-treated rats (14). These findings suggest that FGFR1 inhibition may be an ideal therapeutic approach to HSC activation and liver fibrosis. Nevertheless, it is unclear whether FGFR1 is a potential regulator in LPS-related inflammatory responses and activation of HSCs.

The goal of this study was to determine the role of FGFR1 in LPS-induced inflammation and HSCs activation. Specifically, we used pharmacological and genetic means to inhibit FGFR1 in HSCs. We showed that inhibition of FGFR1 ameliorated LPS induced-NF- $\kappa \mathrm{B}$ activation, inflammatory responses, fibrosis and cell viability in HSCs. Furthermore, 
we found that the LPS/TLR4/c-Src signalling axis appeared to mediate downstream FGFR1 phosphorylation.

\section{Materials and methods}

Reagents. AZD4547 were purchased from Shanghai Kai Yu Pharmatech Technology Co., Ltd. (Shanghai China). LPS, PP2, and TAK-242 were purchased from Sigma-Aldrich (St. Louis, MO, USA). AZD4547, PP2, and TAK-242 were dissolved in DMSO for in vitro experiments. Antibodies against TGF- $\beta$, collagen 1, $\alpha$-SMA, p-c-Src, c-Src, lamin B, and GAPDH were purchased from Santa Cruz Biotechnology, Inc. (Dallas, TX, USA). Antibodies against p-FGFR1, FGFR1, TLR4, TNF- $\alpha$, IL-6, IкB- $\alpha$ and NF- $\kappa$ B P65 were from Cell Signaling Technology, Inc. (Danvers, MA, USA).

Cell culture and treatment. HSCs were isolated from male Sprague-Dawley rats (450-500 g) as described previously (16). Animal care and experimental protocols were approved by the Committee on Animal Care of Zhuji People's Hospital of Zhejiang Province (Zhuji, China; approval no. zjdw2017-008). Briefly, after in situ perfusion of the liver with 2-step pronase-collagenase digestion, HSCs were separated from other nonparenchymal cells by density-gradient centrifugation using OptiPrep (Axis-Shield, 1114542). HSCs were maintained in DMEM containing 10\% FBS, $100 \mathrm{U} / \mathrm{ml}$ penicillin, and $100 \mathrm{mg} / \mathrm{ml}$ streptomycin in a humidified atmosphere of $5 \%$ $\mathrm{CO}_{2}$ at $37^{\circ} \mathrm{C}$. All treatments were initiated $12 \mathrm{~h}$ after isolation unless otherwise indicated. All experiments were repeated at least 3 times.

Measurement of cell viability by MTT assay. Cell viability was assessed by MTT assay. HSCs were plated in 96-well plates at 5,000 cells per well and were then treated with or without LPS for $24 \mathrm{~h}$. After incubation with MTT for $3 \mathrm{~h}$, the reduction of MTT to purple formazan was detected by a microplate reader at $540 \mathrm{~nm}$. Cell viability was calculated as follows: Cell viability $=$ Atreated $/$ Acontrol x 100\%.

siRNA-induced gene silencing. FGFR1 gene silencing in cells was achieved by transfecting cells with siRNA (5'-GCAGCG AUACCACCUACUUTT-3') using LipofectAMINE ${ }^{\text {TM }} 2000$ (Invitrogen; Thermo Fisher Scientific, Inc., Waltham, MA, USA). Knockdown was verified by western blotting (WB).

WB and co-immunoprecipitation. HSCs were lysed, and protein amounts were determined by the Bradford assay (Bio-Rad). Nuclear and cytoplasmic proteins were extracted from HSCs using nuclear and cytoplasmic protein extraction kits (Beyotime Biotech, Nantong, China). Proteins were separated by $10 \%$ SDS-PAGE and were electrotransferred to PVDF membranes. Each membrane was blocked for $1.5 \mathrm{~h}$ with Tris-buffered saline containing $0.05 \%$ Tween-20 and $5 \%$ non-fat milk. PVDF membranes were then incubated with specific primary antibodies. Immunoreactive bands were detected by incubating membranes with horseradish peroxidase-conjugated secondary antibodies and visualisation using enhanced chemiluminescence (Bio-Rad). The amounts of the proteins were analysed using ImageJ analysis software version $1.38 \mathrm{e}$ and were normalised to their respective controls.
For immunoprecipitation studies, extracts were incubated with anti-c-Src-antibody for $4 \mathrm{~h}$ and were then precipitated with protein G-Sepharose beads at $4^{\circ} \mathrm{C}$ overnight. c-Src and FGFR1 levels were detected by immunoblotting using specific antibodies.

$R T-q P C R$. Total RNA was isolated from HSCs using TRIzol (Invitrogen; Thermo Fisher Scientific, Inc.). Reverse transcription and quantitative PCR were carried out using a two-step Platinum SYBR Green qPCR SuperMix-UDG kit (Invitrogen; Thermo Fisher Scientific, Inc.). An Eppendorf Mastercycler (Eppendorf, Hamburg, Germany) was used for qPCR analysis. Primers for genes including TNF- $\alpha$, IL- 6 , collagen I, TGF- $\beta$, $\alpha$-SMA, and $\beta$-actin were obtained from Invitrogen; Thermo Fisher Scientific, Inc. (sequences are listed in Table I). Target mRNA was normalised to $\beta$-actin.

Immunofluorescence cell staining. Cells were fixed with $4 \%$ paraformaldehyde, permeabilised with $0.1 \%$ Triton X-100 and stained. Col-1 and $\alpha$-SMA staining were performed by incubating slides with anti-Col- 1 or anti- $\alpha$-SMA antibody at 1:200 dilution overnight at $4^{\circ} \mathrm{C}$. PE-conjugated secondary antibody (1:200) was used for detection. Cells were counterstained with DAPI nuclear stain. Images were captured (original magnification 400; Nikon, Tokyo, Japan).

Statistical analysis. All data represented 3 independent experiments and were expressed as the means \pm SEM. Statistical analyses were performed using GraphPad Pro. Prism 5.0 (GraphPad Software, Inc., La Jolla, CA, USA). Student t-tests or one-way ANOVAs followed by multiple comparisons tests with Bonferroni corrections were employed to analyse the differences between sets of data. A P-value $<0.05$ was considered to indicate a statistically significant difference.

\section{Results}

Small-molecule FGFR1 inhibitor reversed LPS-induced HSC activation. We used a small-molecule inhibitor, AZD4547, that specifically inhibits FGFR1 activity (Fig. 1A) (17). Freshly isolated HSCs were treated with LPS (100 $\mathrm{ng} / \mathrm{ml}$ for $15 \mathrm{~min})$ and the effects on FGFR1 activation were determined. LPS induced a robust increase in FGFR1 phosphorylation that was inhibited in a dose-dependent fashion by AZD4547 pretreatment for $1 \mathrm{~h}$ (Fig. 1B). To evaluate the effect of AZD4547 on LPS-induced HSCs activation, we determined cell viability of HSCs. In accordance with previous studies (18), LPS significantly stimulated HSC proliferation (Fig. 1C), indicating that LPS increased HSC activation. Treatment with AZD4547 reduced LPS-induced cell viability (Fig. 1C).

Increased production and/or activity of transforming growth factor (TGF)- $\beta$ was critical for sustaining HSC activation and fibrosis (19). Upon sustained LPS treatment for $6 \mathrm{~h}$, mRNA levels of TGF- $\beta$ increased (Fig. 1D). Pretreatment with AZD4547 decreased TGF- $\beta$ mRNA levels in a dose-dependent manner (Fig. 1D). As shown in Fig. 1D, LPS stimulated mRNA expression of ECM, including collagen I and $\alpha$-smooth muscle actin $(\alpha$-SMA), both of which were reduced by AZD4547 pretreatment in a dose-dependent manner. AZD4547 also dose-dependently reversed LPS-stimulated TGF- $\beta$ (Fig. 1E), 
Table I. Sequences of primers for RT-qPCR assay used in the study.

\begin{tabular}{lccc}
\hline Gene & Species & Forward primer & \multicolumn{1}{c}{ Reverse primer } \\
\hline TNF- $\alpha$ & Rat & 5'-TACTCCCAGGTTCTCTTCAAGG-3' & 5'-GGAGGCTGACTTTCTCCTGGTA-3' \\
IL-6 & Rat & 5'-GAGTTGTGCAATGGCAATTC-3' & 5'-ACTCCAGAAGACCAGAGCAG-3' \\
Collagen 1 & Rat & 5'-CGAGTATGGAAGCGAAGGTT-3' & 5'-ACGCTGTTCTTGCAGTGATA-3' \\
TGF- $\beta$ & Rat & 5'-AGGAGGAATTTGGCCAGGTG-3' & 5'-GCTCACGAGGAGGCTAATCC-3' \\
$\alpha-S M A$ & Rat & 5'-TGACCCAGATTATGTTTGAG-3' & 5'-AGATAGGCACGTTGTGAGTC-3' \\
$\beta$-actin & Rat & 5'-AAGTCCCTCACCCTCCCAAAAG-3' & 5'-AAGCAATGCTGTCACCTTCCC-3' \\
\hline
\end{tabular}

A

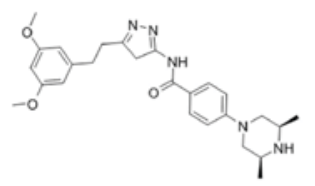

AZD4547

T-FGFR1 inhibition:IC50 $=0.2 \mathrm{nM}$
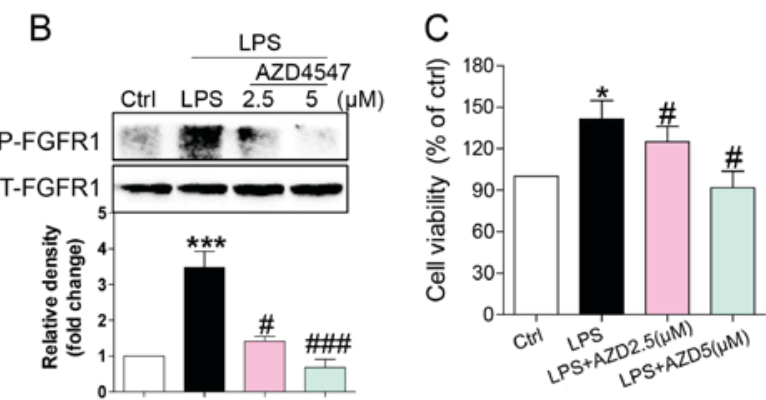

D

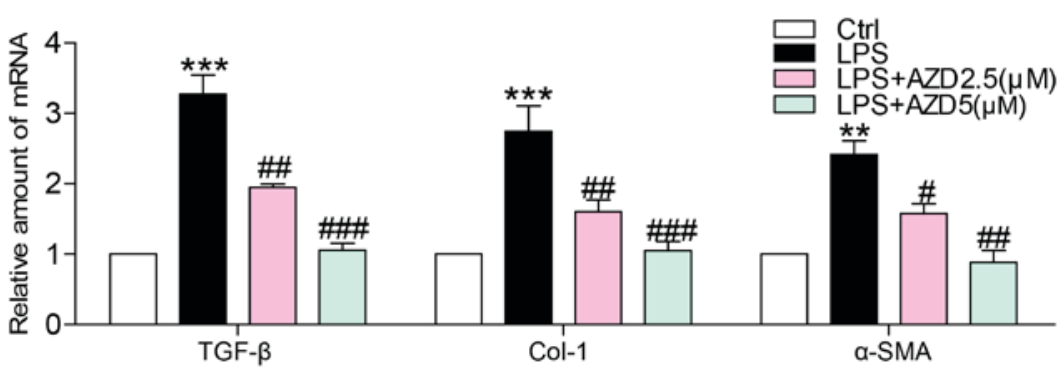

$\mathrm{E}$
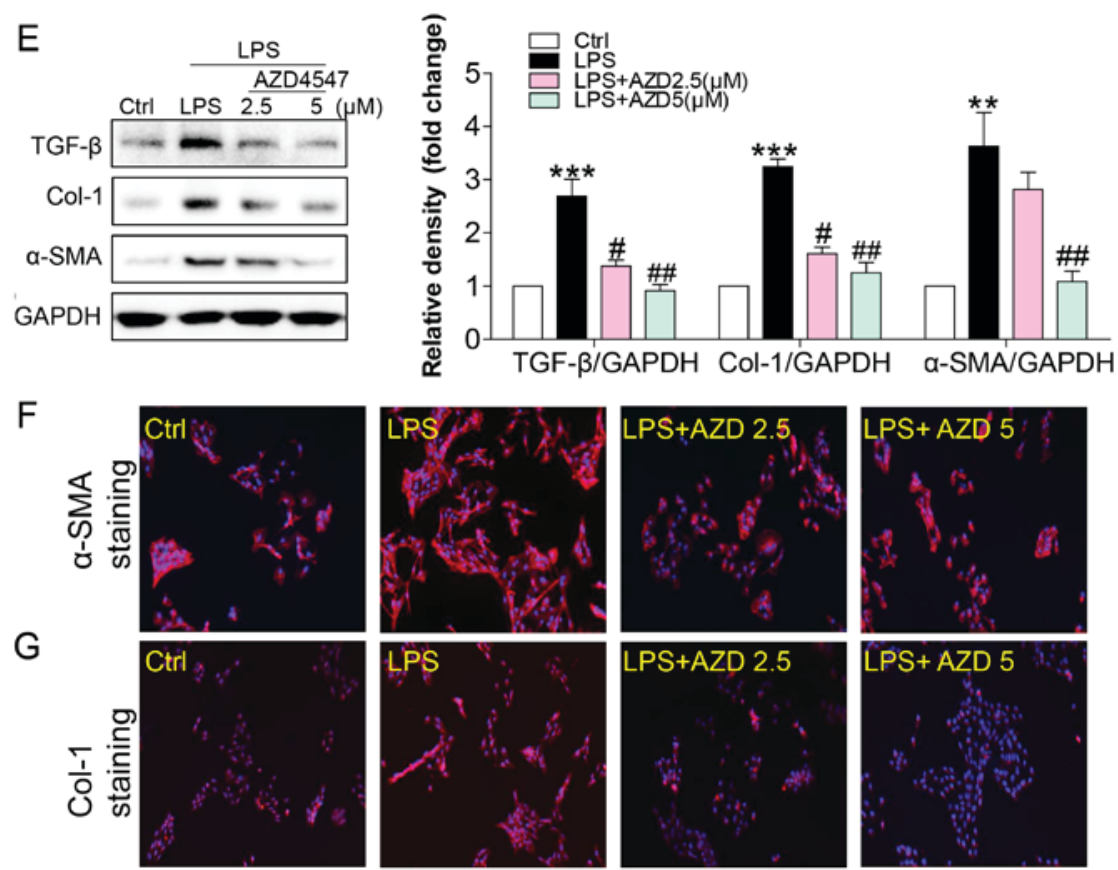

Figure 1. FGFR1 inhibitor AZD4547 attenuates LPS-induced HSCs activation. (A) Chemical structures and FGFR1-inhibitory IC50 values of AZD4547. HSCs were pretreated with AZD4547 (AZD, 2.5, $5 \mu \mathrm{M}$ ) for $1 \mathrm{~h}$, and then exposed to LPS (100 ng/ml) for the indicated times. (B) Exposure to LPS for $15 \mathrm{~min}$. p-FGFR1 levels detected by western blotting (WB). (C) Exposure to LPS for $24 \mathrm{~h}$. The cell viability of HSCs detected by MTT assay. (D) Exposure to LPS for $6 \mathrm{~h}$, the mRNA levels of TGF- $\beta$, col-1, and $\alpha$-SMA were detected by RT-qPCR and normalised by $\beta$-actin. Incubation with LPS for $24 \mathrm{~h}$. (E) The levels of TGF- $\beta$, Col-1 and $\alpha$-SMA in cell lysates were detected by WB. (F and G) Immunofluorescence staining of HSCs for Col-1 (red), and $\alpha$-SMA (red) in LPS-treated cells pretreated with AZD4547 prior to LPS exposure (blue=DAPI). Representative micrographs are shown $\left({ }^{*} \mathrm{P}<0.05,{ }^{* *} \mathrm{P}<0.01,{ }^{* * *} \mathrm{P}<0.001, \mathrm{vs}\right.$. Ctrl group; ${ }^{\#} \mathrm{P}<0.05,{ }^{\# \#} \mathrm{P}<0.01,{ }^{\# \# \#} \mathrm{P}<0.001$, vs. LPS group). 
A

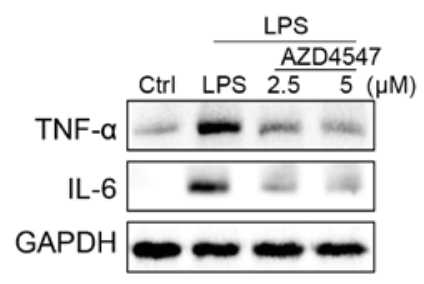

C

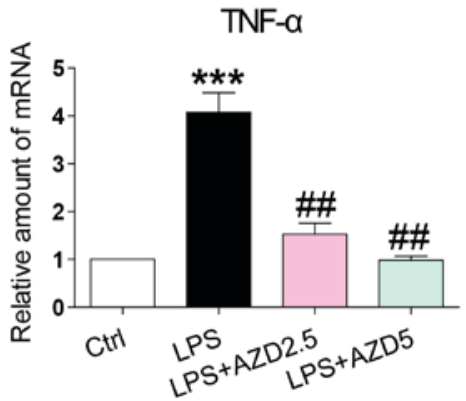

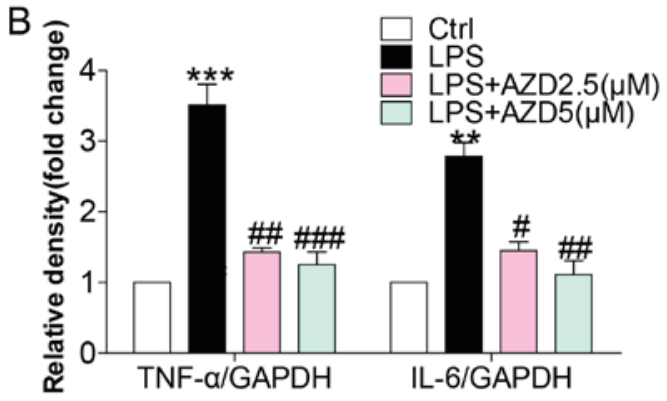

D

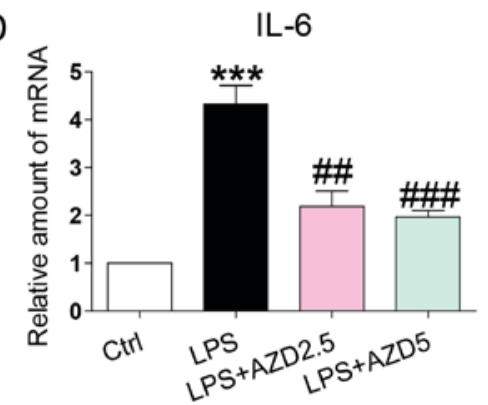

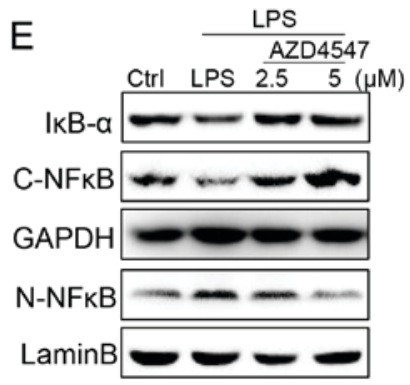

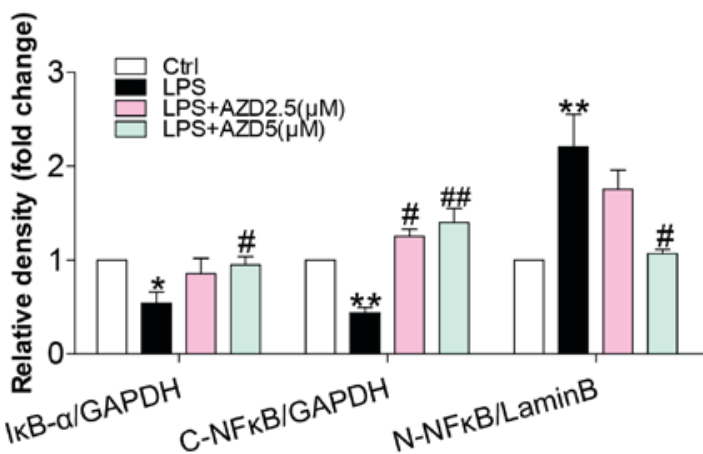

Figure 2. AZD4547 attenuates LPS-induced inflammatory response in HSCs. HSCs were pretreated with AZD4547 (AZD, 2.5, $5 \mu \mathrm{M}$ ) for $1 \mathrm{~h}$, and then exposed to LPS $(100 \mathrm{ng} / \mathrm{ml})$ for indicated times. (A) Incubation with LPS for $24 \mathrm{~h}$. TNF- $\alpha$ and IL-6 in cell lysates were detected by western blotting (WB). (B) The column figures show the normalised optical density for the data from three independent experiments. Incubation with LPS for $6 \mathrm{~h}$ was indicated. The mRNA

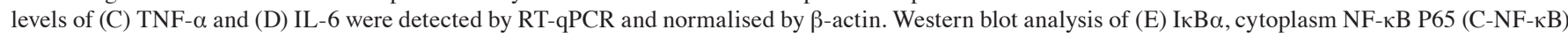

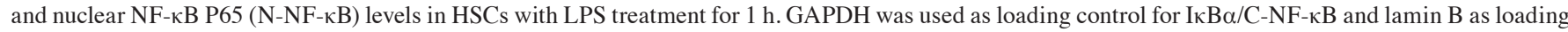
control for N-NF-kB (ns=not significant vs. Ctrl group; ${ }^{* * *} \mathrm{P}<0.01,{ }^{* * *} \mathrm{P}<0.001$, vs. Ctrl group; ${ }^{*} \mathrm{P}<0.05,{ }^{, \#} \mathrm{P}<0.01,{ }^{\# \# \#} \mathrm{P}<0.001$, vs. LPS group).

Col-1 (Fig. 1E), and $\alpha$-SMA (Fig. 1E) protein expression. These results were also verified by staining cells for $\alpha$-SMA (Fig. 1F), and Col-1 (Fig. 1G). These findings strongly suggested that the FGFR1 small-molecule inhibitor attenuated LPS-related fibrosis in HSCs, and that inhibition of liver fibrosis protein expression by AZD4547 may be associated with decreased HSC viability.

FGFR1 inhibitor AZD4547 decreased LPS-induced inflammatory responses in HSCs. LPS caused inflammatory responses in the liver, mediating the progression of HSC activation $(8,10,20)$. We evaluated whether AZD4547 altered the expression of pro-inflammatory cytokines. Immunoblotting showed an increased expression of inflammatory cytokines, including tumour necrosis factor- $\alpha$ (TNF- $\alpha$ ) and interleukin-6 (IL-6) in HSCs (Fig. 2A and B). This increase was associated with increased mRNA levels of TNF- $\alpha$ and IL-6 (Fig. 2C and D). AZD4547 treatment reduced both protein and mRNA levels of TNF- $\alpha$ and IL-6 (Fig. 2A-D). To uncover the signalling mechanism underlying the anti-inflammatory activity of AZD4547, we examined the NF- $\mathrm{KB}$ pathway, the signalling pathway implicated in the expression of pro-inflammatory cytokines by LPS in HSCs (10). We exposed HSCs to LPS and treated the cells with AZD4547 to assess the NF- $\kappa B$ signalling pathway. LPS reduced cytosolic IкB- $\alpha$ (Fig. 2E), cytoplasmic NF- $\kappa$ B p65 subunit (Fig. 2E) and increased nuclear NF-кB p65 subunit (Fig. 2E) levels. AZD4547 treatment of HSCs prevented LPS-induced reduction in cytosolic IкB- $\alpha$, p65 and increased nuclear p65 levels (Fig. 2E). These results show that LPS induced a pro-inflammatory phenotype in HSCs and that these adverse effects were prevented by FGFR1 inhibitor AZD4547.

Gene knockdown of FGFRI attenuated LPS-induced inflammatory responses and activation of HSCs. To assess the non-specificity of the small molecule inhibitor and to provide further support for the role of FGFR1, we silenced FGFR1 by siRNA (si-FGFR1). Transfection of si-FGFR1 led 
A
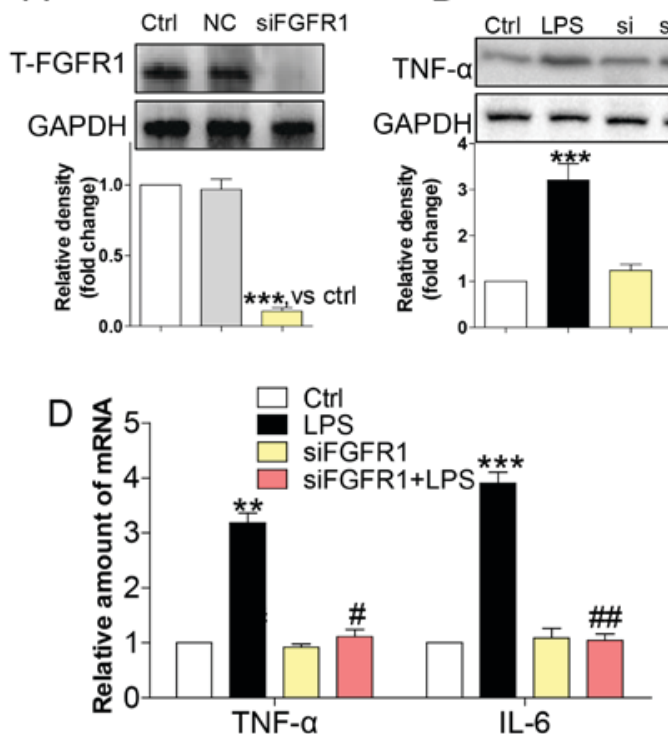

C

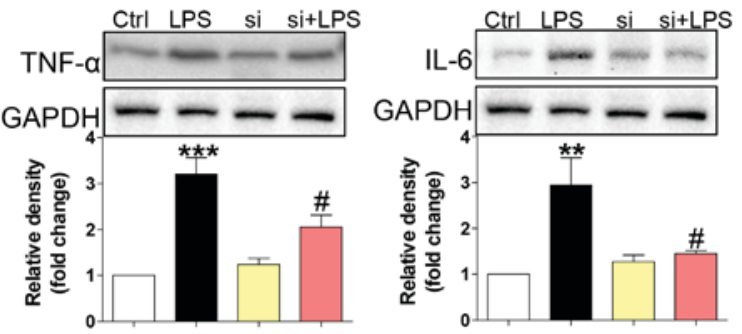

$\mathrm{E}$

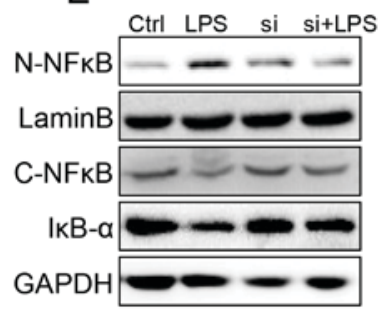

$\mathrm{F}$

G
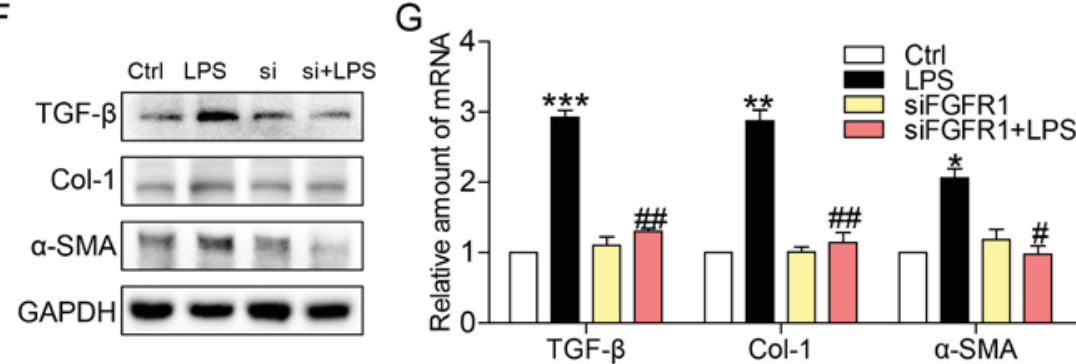

Figure 3. siFGFR1 decreases LPS-induced HSCs injury. (A) Western blot analysis of FGFR1 following siRNA transfection in HSCs (NC=negative control transfection). After incubating for $24 \mathrm{~h}$, FGFR1 knockdown HSCs were stimulated with LPS (100 ng/ml) for indicated times (Si=FGFR1 siRNA). Cells were incubated with LPS for 24 h. (B) TNF- $\alpha$ and (C) IL-6 in cell lysates were detected by western blotting (WB). (D) Incubated with LPS for 6 h. The mRNA levels of TNF- $\alpha$ and IL- 6 were detected by RT-qPCR and normalised by $\beta$-actin. (E) I $\kappa$ B- $\alpha, \mathrm{C}-\mathrm{NF}-\kappa \mathrm{B}$, and N-NF- $\kappa \mathrm{B}$ protein levels were determined in cells with LPS treatment for $1 \mathrm{~h}$. (F) Incubated with LPS for $24 \mathrm{~h}$. The levels of TGF- $\beta$, col-1, and $\alpha$-SMA were detected by WB. (G) Exposing to LPS for $6 \mathrm{~h}$, the mRNA levels of TGF- $\beta$, col- 1 , and $\alpha$-SMA were detected by RT-qPCR and normalised by $\beta$-actin $\left({ }^{*} \mathrm{P}<0.05\right.$, ${ }^{* *} \mathrm{P}<0.01,{ }^{* * *} \mathrm{P}<0.001$, vs. Ctrl group; ${ }^{*} \mathrm{P}<0.05$, ${ }^{\# \#} \mathrm{P}<0.01$, vs. LPS group).

to decreased FGFR1 protein expression in HSCs (Fig. 3A), and attenuated protein (Fig. 3B and C) and gene (Fig. 3D) expression levels of TNF- $\alpha$ and IL-6 in LPS-stimulated HSCs. In addition, LPS-induced NF- $\mathrm{kB}$ activity was not evident following silencing of FGFR1 expression in HSCs (Fig 3E). Subsequently, si-FGFR1 remarkably decreased LPS-induced activation of HSCs, as evidenced by liver fibrosis markers such as TGF- $\beta$, col- 1 , and $\alpha$-SMA at both the protein (Fig. 3F) and mRNA (Fig. 3G) level. These findings, together with the results of the anti-inflammation and anti-fibrosis effect of AZD4547, confirmed that FGFR1 had a potential role in regulating LPS-related HSCs activation.

LPS triggered FGFR1 phosphorylation in HSCs through the TLR4/c-Src signalling cascade. How LPS activates FGFR1 remained unaddressed. LPS directly binds to TLR4 (9), subsequently activating downstream NF- $\mathrm{\kappa B}$ signalling and inflammatory responses $(21,22)$. TLR4 signalling activation promoted c-Src phosphorylation (23). It was also reported that $\mathrm{c}$-Src via FGFR1 transactivation and subsequent downstream activation of multiple pathways mediated lymphoma and myeloproliferative disorders (24). Therefore, we tested whether LPS activated FGFR1 in HSCs through the TLR4/c-Src signalling cascade. AZD4547 did not block expression of TLR4 (Fig. 4A) or phosphorylation of c-Src (Fig. 4B) induced by LPS, suggesting that FGFR1 may be not be the upstream regulator of TLR4/c-Src. We then evaluated the role of TLR4 blocker TAK-242 and c-Src blocker PP2 in LPS induced-FGFR1 phosphorylation. As shown in Fig. 4C, both TAK-242 and PP2 reduced FGFR1 activation in HSCs. In addition, gene knockdown of FGFR1 did not suppress LPS-induced increases in TLR4 (Fig. 4D) or activation of c-Src (Fig. 4E). We also assessed c-Src/FGFR1 complex formation in the context of LPS. Co-immunoprecipitation showed that LPS challenge of HSCs for 5 to 30 min remarkably increased the recruitment of FGFR1 to c-Src (Fig. 4F). These findings suggested a novel mechanism of LPS-induced FGFR1 activation, one that involves TLR4/c-Src signalling cascade.

\section{Discussion}

In this study, we revealed that LPS mediated FGFR1 activation in HSCs, which then contributed to NF- $\mathrm{KB}$ activation, IL-6 and TNF- $\alpha$ release, fibrosis and proliferation in HSCs. Application of an FGFR1 inhibitor or genetic knockdown of FGFR1 in LPS-challenged cells produced a great reduction in 

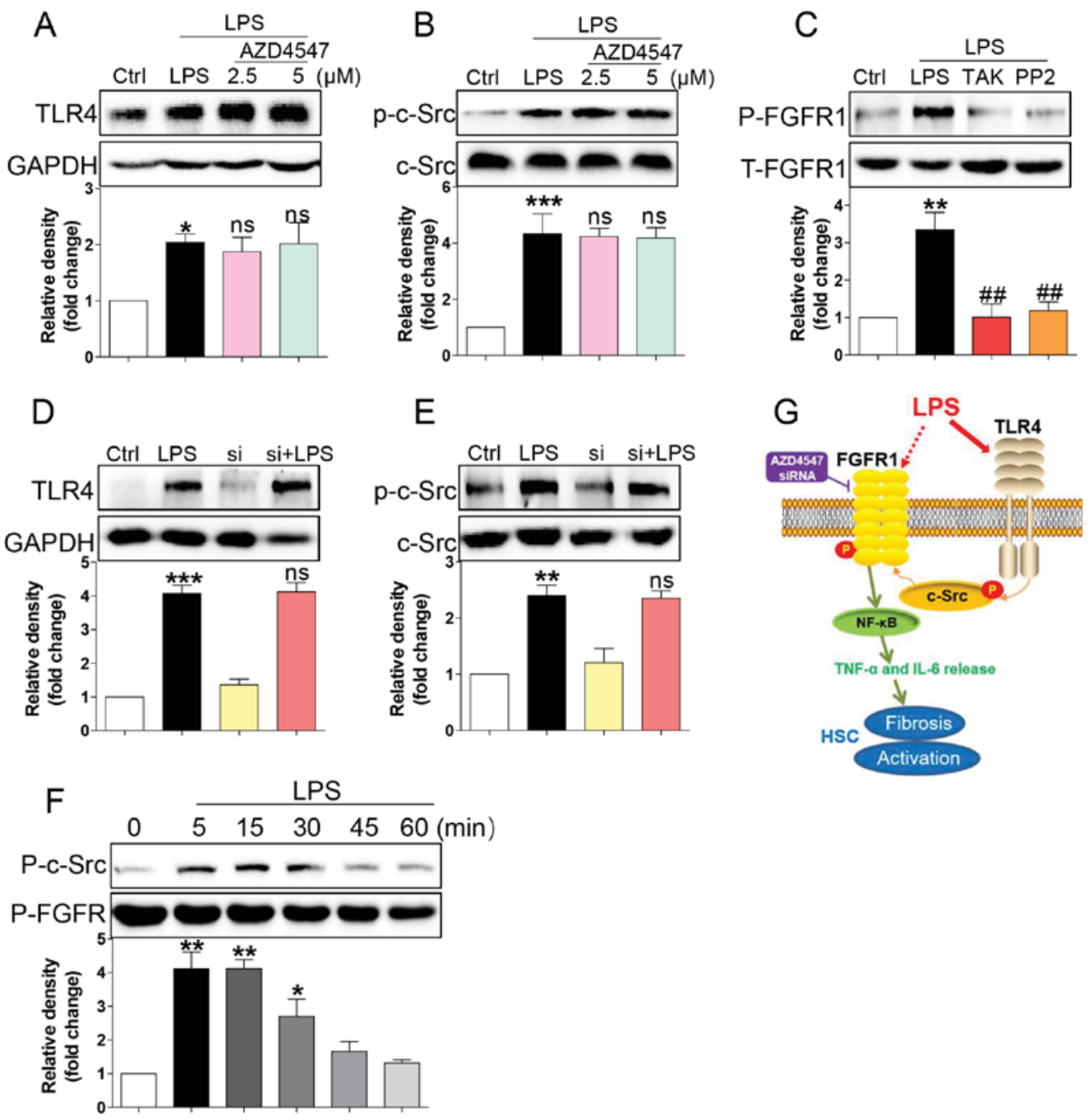

Figure 4. LPS triggers FGFR1 phosphorylation in HSCs through the TLR4/c-Src signalling cascade. HSCs were pre-treated with AZD4547 (AZD, 2.5 , $5 \mu \mathrm{M}$ ) for $1 \mathrm{~h}$. After LPS treatment for $15 \mathrm{~min}$, the expression of (A) TLR4 and (B) phosphorylation levels of c-Src were detected by western blotting (WB). (C) HSCs were pre-treated with TAK-242 (TLR4 inhibitor), or PP2 (c-Src inhibitor) for $1 \mathrm{~h}$, followed by LPS treatment for 15 min. Phosphorylation of FGFR1 was determined via WB. (D and E) siFGFR1 did not reduce LPS (15 min)-induced increase expression of TLR4 and c-Src activation. (F) LPS-induced interaction between c-Src and FGFR1. HSCs were exposed to LPS for the indicated times. Lysates were then subjected to c-Src IP and FGFR1 was measured. (G) Schematic illustration of the major findings of this study: LPS activated TLR4 and increased signalling through the c-Src pathway to cause FGFR1 activation and production of inflammatory cytokines. This proinflammatory response produced HSCs activation and liver fibrosis $\left({ }^{*} \mathrm{P}<0.05,{ }^{* * *} \mathrm{P}<0.01,{ }^{* * * *} \mathrm{P}<0.001, \mathrm{vs}\right.$. Ctrl group; ns, not significant vs. LPS group; ${ }^{\# \#} \mathrm{P}<0.01$, vs. LPS group).

HSC viability, fibrosis and inflammatory responses, suggesting that inhibition of FGFR1 may be a therapeutic approach for LPS-induced HSC activation (Fig. 4G).

Evidence implicated FGFR1 in a host of liver fibrosis diseases (13-15). Böhm et al (13) generated mice with hepatocytes that lacked FGFR1 and subjected them to acute and chronic CCl4-induced liver injury and partial hepatectomy. In hepatocytes, loss of FGFR1 eliminated responsiveness to FGF7 but did not affect toxin-induced liver injury and fibrosis. However, mortality after partial hepatectomy increased because of severe hepatocyte necrosis (13). Using a tissue microarray of 89 primary liver tumours, including a subset of 10 fibrolamellar carcinomas, Riehle et al (15) provided evidence of FGFR1 overexpression in human fibrolamellar carcinoma and supported the use of FGFR1 inhibitors in the treatment of patients with unresectable fibrolamellar carcinoma. Our results indicated that FGFR1 inhibitor or genetic silencing by siRNA significantly decreased the expression of
ECMs, including TGF- $\beta, \alpha-$ SMA, collagen I, and reduced cell viability in HSCs related to LPS.

The NF- $\mathrm{BB}$ signalling pathway, a conserved mediator of inflammatory responses, plays a central role in regulating the progression of liver fibrogenesis (25). Inhibition of I $\mathrm{B}$ B kinases stimulated HSC apoptosis, indicating that NF- $\kappa \mathrm{B}$ signalling played a central role in the activation of HSC (10). Liver fibrosis is often associated with LPS-induced pro-inflammatory cytokines IL- 6 , and TNF- $\alpha$ release (26). We showed that the same FGFR1/NF- $\mathrm{B}$ activation pathway enhanced inflammatory responses in HSCs that were markedly reversed by the FGFR1 inhibitor AZD4547 or siRNA-silencing FGFR1.

There is a pressing need to understand how LPS activates FGFR1 signalling. Yao et al (27) found that dioscin exhibited protective effects against LPS-induced liver injury via altering TLR4 signalling. We identified TLR4, the classic receptor for LPS, as a potential activator of FGFR1 in HSCs. In HFD-fed mice, TLR4 and FGFR1 appeared to be implicated 
in the expression of proinflammatory cytokines and hepatic steatosis (28). It has been reported that Src activation played an important key role in FGFR1 kinase activation $(24,29)$. A.E. Medvedev and colleagues found that c-Src kinase played a key role in LPS-dependent NF- $\kappa \mathrm{B}$ activation (30). Here, we identified for the first time that TLR4 interacted with LPS to facilitate c-Src/FGFR1 interactions, thereby activating downstream NF- $\kappa$ B signalling and inflammatory responses (Fig. 4F).

In summary, we demonstrated that FGFR 1 mediated LPS-induced $\mathrm{NF}-\kappa \mathrm{B}$ activation, inflammatory responses and activation of HSCs. LPS-induced FGFR1 activation appeared to require upstream TLR4-Src-related mechanisms. Our data suggested that FGFR1 inhibition may be a feasible strategy for treating LPS-related liver fibrosis.

\section{Acknowledgements}

Not applicable.

\section{Funding}

The project supported by research grants from the Zhejiang Provincial Program of Chinese Medical and Health Science Funding (grant no. 20172A141), Zhejiang Provincial Program of Medical and Health Science Funding (grant no. 2017KY679), Zhuji City Natural Science Funding and Zhejiang Pharmaceutical Association Science Funding (grant no. 2016ZYY30).

\section{Availability of data and materials}

The datasets used and/or analyzed during the current study are available from the corresponding author on reasonable request.

\section{Authors' contributions}

DL, JH, and LZ performed the experiments. YQ, JH, DL and LJ designed the research study. YQ and LJ contributed essential reagents or tools. HM, JX, JS, LJ and ZX analysed the data. $\mathrm{LJ}$ and $\mathrm{JH}$ wrote the paper.

\section{Ethics approval and consent to participate}

Animal care and experimental protocols were approved by the Committee on Animal Care of Zhuji People's Hospital of Zhejiang Province (Zhuji, China; approval no. zjdw2017-008).

\section{Patient consent for publication}

Not applicable.

\section{Competing interests}

The authors declare that they have no competing interests.

\section{References}

1. Koyama $\mathrm{Y}$ and Brenner DA: Liver inflammation and fibrosis J Clin Invest 127: 55-64, 2017.
2. Schuppan D and Kim YO: Evolving therapies for liver fibrosis. J Clin Invest 123: 1887-1901, 2013.

3. Gu L, Tao X, Xu Y, Han X, Qi Y, Xu L, Yin L and Peng J: Dioscin alleviates BDL- and DMN-induced hepatic fibrosis via Sirt1/Nrf2-mediated inhibition of p38 MAPK pathway. Toxicol Appl Pharmacol 292: 19-29, 2016.

4. Xu L, Yin L, Tao X, Qi Y, Han X, Xu Y, Song S, Li L, Sun P and Peng J: Dioscin, a potent ITGA5 inhibitor, reduces the synthesis of collagen against liver fibrosis: Insights from SILAC-based proteomics analysis. Food Chem Toxicol 107: 318-328, 2017.

5. Zhang X, Han X, Yin L, Xu L, Qi Y, Xu Y, Sun H, Lin Y, Liu K and Peng J: Potent effects of dioscin against liver fibrosis. Sci Rep 5: 9713, 2015.

6. Yin C, Evason KJ, Asahina K and Stainier DY: Hepatic stellate cells in liver development, regeneration, and cancer. J Clin Invest 123: 1902-1910, 2013.

7. Yin L, Qi Y, Xu Y, Xu L, Han X, Tao X, Song S and Peng J: Dioscin inhibits HSC-T6 cell migration via adjusting SDC-4 expression: Insights from iTRAQ-based quantitative proteomics. Front Pharmacol 8: 665, 2017.

8. Fouts DE, Torralba M, Nelson KE, Brenner DA and Schnabl B: Bacterial translocation and changes in the intestinal microbiome in mouse models of liver disease. J Hepatol 56: 1283-1292, 2012.

9. Hedayat M, Netea MG and Rezaei N: Targeting of Toll-like receptors: A decade of progress in combating infectious diseases. Lancet Infect Dis 11: 702-712, 2011.

10. Oakley F, Meso M, Iredale JP, Green K, Marek CJ, Zhou X, May MJ, Millward-Sadler H, Wright MC and Mann DA: Inhibition of inhibitor of kappaB kinases stimulates hepatic stellate cell apoptosis and accelerated recovery from rat liver fibrosis. Gastroenterology 128: 108-120, 2005.

11. Mohammadi M, Olsen SK and Ibrahimi OA: Structural basis for fibroblast growth factor receptor activation. Cytokine Growth Factor Rev 16: 107-137, 2005.

12. Fischer H, Taylor N, Allerstorfer S, Grusch M, Sonvilla G, Holzmann K, Setinek U, Elbling L, Cantonati H, Grasl-Kraupp B, et al: Fibroblast growth factor receptor-mediated signals contribute to the malignant phenotype of non-small cell lung cancer cells: Therapeutic implications and synergism with epidermal growth factor receptor inhibition. Mol Cancer Ther 7: 3408-3419, 2008.

13. Böhm F, Speicher T, Hellerbrand C, Dickson C, Partanen JM, Ornitz DM and Werner S: FGF receptors 1 and 2 control chemically induced injury and compound detoxification in regenerating livers of mice. Gastroenterology 139: 1385-1396, 2010.

14. Lin N, Chen S, Pan W, Xu L, Hu K and Xu R: NP603, a novel and potent inhibitor of FGFR1 tyrosine kinase, inhibits hepatic stellate cell proliferation and ameliorates hepatic fibrosis in rats. Am J Physiol Cell Physiol 301: C469-C477, 2011.

15. Riehle KJ, Yeh MM, Yu JJ, Kenerson HL, Harris WP, Park JO and Yeung RS: mTORC1 and FGFR1 signaling in fibrolamellar hepatocellular carcinoma. Mod Pathol 28: 103-110, 2015.

16. Mederacke I, Dapito DH, Affò S, Uchinami H and Schwabe RF: High-yield and high-purity isolation of hepatic stellate cells from normal and fibrotic mouse livers. Nat Protoc 10: 305-315, 2015.

17. Tucker JA, Klein T, Breed J, Breeze AL, Overman R, Phillips C and Norman RA: Structural insights into FGFR kinase isoform selectivity: Diverse binding modes of AZD4547 and ponatinib in complex with FGFR1 and FGFR4. Structure 22: 1764-1774, 2014.

18. Liu M, Xu Y, Han X, Yin L, Xu L, Qi Y, Zhao Y, Liu K and Peng J: Dioscin alleviates alcoholic liver fibrosis by attenuating hepatic stellate cell activation via the TLR4/MyD88/NF- $\mathrm{B}$ signaling pathway. Sci Rep 5: 18038, 2015.

19. Pinzani M and Marra F: Cytokine receptors and signaling in hepatic stellate cells. Semin Liver Dis 21: 397-416, 2001.

20. Ceccarelli S, Panera N, Mina M, Gnani D, De Stefanis C, Crudele A, Rychlicki C, Petrini S, Bruscalupi G, Agostinelli L, et al: LPS-induced TNF- $\alpha$ factor mediates pro-inflammatory and pro-fibrogenic pattern in non-alcoholic fatty liver disease. Oncotarget 6: 41434-41452, 2015.

21. Qi M, Yin L, Xu L, Tao X, Qi Y, Han X, Wang C, Xu Y, Sun H, Liu K aned Peng J: Dioscin alleviates lipopolysaccharide-induced inflammatory kidney injury via the microRNA let-7i/TLR4/MyD88 signaling pathway. Pharmacol Res 111: 509-522, 2016.

22. Qi M, Zheng L, Qi Y, Han X, Xu Y, Xu L, Yin L, Wang C, Zhao Y, Sun H, et al: Dioscin attenuates renal ischemia/reperfusion injury by inhibiting the TLR4/MyD88 signaling pathway via up-regulation of HSP70. Pharmacol Res 100: 341-352, 2015. 
23. Shan X, Zhang Y, Chen H, Dong L, Wu B, Xu T, Hu J, Liu Z, Wang W, Wu L, et al: Inhibition of epidermal growth factor receptor attenuates LPS-induced inflammation and acute lung injury in rats. Oncotarget 8: 26648-26661, 2017.

24. Ren M, Qin H, Ren R, Tidwell J and Cowell JK: Src activation plays an important key role in lymphomagenesis induced by FGFR1 fusion kinases. Cancer Res 71: 7312-7322, 2011.

25. Xiao C and Ghosh S: NF-kappaB, an evolutionarily conserved mediator of immune and inflammatory responses. Adv Exp Med Biol 560: 41-45, 2005.

26. Paik YH, Schwabe RF, Bataller R, Russo MP, Jobin C and Brenner DA: Toll-like receptor 4 mediates inflammatory signaling by bacterial lipopolysaccharide in human hepatic stellate cells. Hepatology 37: 1043-1055, 2003.

27. Yao H, Hu C, Yin L, Tao X, Xu L, Qi Y, Han X, Xu Y, Zhao Y Wang $C$ and Peng J: Dioscin reduces lipopolysaccharide-induced inflammatory liver injury via regulating TLR4/MyD88 signal pathway. Int Immunopharmacol 36: 132-141, 2016.
28. Park S, Choi Y, Um SJ, Yoon SK and Park T: Oleuropein attenuates hepatic steatosis induced by high-fat diet in mice. J Hepatol 54: 984-993, 2011.

29. Zou L, Cao S, Kang N, Huebert RC and Shah VH: Fibronectin induces endothelial cell migration through $\beta 1$ integrin and Src-dependent phosphorylation of fibroblast growth factor receptor-1 at tyrosines $653 / 654$ and 766 . J Biol Chem 287: 7190-7202, 2012.

30. Medvedev AE, Piao W, Shoenfelt J, Rhee SH, Chen H, Basu S, Wahl LM, Fenton MJ and Vogel SN: Role of TLR4 tyrosine phosphorylation in signal transduction and endotoxin tolerance. J Biol Chem 282: 16042-16053, 2007.

(c) (i) (9) This work is licensed under a Creative Commons A Attribution-NonCommercial-NoDerivatives 4.0 International (CC BY-NC-ND 4.0) License. 